\title{
REVIEWING THE THEORIES OF MOTION OF THE SATELLITES OF SATURN
}

\author{
L. DURIEZ \\ Laboratoire d'Astronomie de l'Université de Lille \\ 1, Impasse de l'Observatoire, F 59000 Lille, France
}

\begin{abstract}
The state of knowledge of the motions of all Saturn's satellites is presented (excluding however the rings and their relating shepherding satellites). In particular, it appears that the theory of motion of the major satellites is now more precise than the available Earth-based observations, allowing to expect new progress with the next observations from mutual events and then with those from the Cassini mission.
\end{abstract}

\section{Introduction}

The saturnian satellite system is certainly the richest and the most complex in the solar system, specially because of the presence of many resonances: There are 3 resonances between 6 of the major satellites (resonance 1:2 between Mimas and Tethys and between Enceladus and Dione, and resonance 3:4 between Titan and Hyperion), 1 resonance 1:1 between the 2 small coorbitals Janus and Epimetheus, and 3 resonances 1:1 between Tethys and its 2 Lagrangian companions Telesto and Calypso and between Dione and its companion Helena. Then, these Lagrangian satellites are also in resonance 1:2 with Mimas and Enceladus respectively. This resonant feature is unique in the solar system. This present status may have been driven by tidal dissipation (by modifying both mean motions and precession rates of nodes and pericentres) and then locked on the observed resonances. According to Dermott et al. (1988), in comparison with the uranian satellites system where no resonance exists now, the resonances in the saturnian system seem to be more regular and more stable because of the larger oblateness of Saturn.

But, before describing the real story of the system, it is important to know accurately the present status of the motions, specially because of spa- 
tial observations of the Saturn's satellites from the past missions (Pioneer and Voyager) or from the planed Cassini mission, it will be necessary to have theories of motions as accurate as a few kilometers.

Hence, because of the lack of place in the proceedings, the present review tries to emphasize only the most recent developments of theories and of observations in the restricted field of the major saturnian satellites. However, an extended full $\mathrm{T}_{\mathrm{E}} \mathrm{X}$ version of this review is available on ask from the author by e-mail at : duriez@gat.univ-lille1.fr; it includes a review of both major and small satellites, some historical aspects and an extended bibliography.

\section{The recent works about major satellites}

All recent works about modelling the motions of the major satellites attempt to reduce the residuals at all costs, attacking the problem of improving both observations and theories (by numerical and analytical methods as well).

\subsection{OBSERVATIONS}

Strugnel \& Taylor (1990) have compiled a catalogue of 51000 ground-based observations made between 1874 and 1989, all being now given in the B1950 reference frame, all times being reduced to UTC only. The quality of each observation is indicated by $(o-c)$ computed from the theories quoted in Taylor \& Shen (1988) but using the mean motions and the libration parameters obtained by Dourneau (1987). The catalogue contains most of the published observations made since 1966 (mainly photographic ones) while other data concern a selection of older, mostly visual, observations. To examine the possibility of combining old and recent observations, Harper et al. (1989) have analyzed the micrometric observations of Titan, Hyperion and Iapetus made from 1874 to 1923 , fitting them to a numerical integration of their motions. The method is the same as that used by Sinclair \& Taylor (1985) with the 1967-1985 observations of these satellites. It results in particular that visual observations of angular separation from the Saturn's centre are rather prone to error (systematically large by about 0 "..5). However the order of magnitude of residuals is found to be the same for visual intersatellite observations and for astrometric observations analyzed by Sinclair \& Taylor, allowing to plan a next fitting of all the data set. It is hoped that future observations be published in the standardized form given by Strugnel \& Taylor.

Other works show that observations are now revitalized by new techniques: improvement of astrographic measurements, emergence of promis- 
ing CCD observations and opportunity of photometric observations of mutual events during the 1995-1996 opposition.

According to Veillet et al. (1988), by using refined astrographic techniques, the precision of the best ground-based observations may be estimated at about $0^{\prime \prime} .05$, that is about $350 \mathrm{~km}$ on positions in the saturnian system. Following the same approach as Sinclair $(1974,1977)$ to derive corrections to the ephemeris of Saturn as well as corrections of the orbits of the satellites, Pascu \& Schmidt (1990) have reduced again their photographic observations of the Saturn's satellites since 1974. They have shown that these observations have an expected precision better than $0^{\prime \prime} .2$ which would suffice to give, as a by-product, better positions of Saturn than by transit circle observations which are not more accurate than 0 " .5 . The same improvement of the Saturn ephemeris is also deduced by Taylor et al. (1991) from Carlsberg and Bordeaux meridian circle observations of Titan and Iapetus made in 1987 and 1989.

Besides, many observers are now ready to use CCD cameras to obtain images of the Saturn's satellites. In spite of the smallness of the field, astrometric measurements on these images could be now more accurate than large field photographic astrometry: Le Floch (1995) describes the technique used at Bordeaux observatory to obtain astrometric positions of major satellites on CCD fields, using the globular cluster M15 to calibrate the images (a catalogue of hundreds of stars in this cluster have been elaborated by Le Campion et al. (1992) specially for small field astrometry); the precision of the reduction of CCD images of M15 is $0^{\prime \prime} .025$ and that of satellite's images may reach the same value. However, Beurle et al. (1993), Beurle (1994) and Harper (1995) describe an analogous work made at La Palma Observatory, but they predict an accuracy of only 0 "'1 on inter-satellite positions. In fact, Pascu (1995) thinks that CCD astrometric observations must be made for faint satellites only, because small fields on CCD chips do not allow to determine the orientation with the same precision as on photographic plates; moreover, if used in astrometric measures, Pascu thinks that the CCD chip must be calibrated in the same manner as the plate-measuring machines (size, position and alignment of each pixel on the chip). Nevertheless, the results obtained by Rapaport \& Le Campion (1995) in fitting theories to CCD inter-satellite positions made at Bordeaux and calibrated with M15, show r.m.s. residuals between 0 ".06 and 0 ".08, that is almost as good results as with the best photographic observations.

At last, observations of mutual events between the Saturn's satellites are planed in 1995-1996. Arlot \& Thuillot (1993), Aksnes \& Dourneau (1994) and Emel'yanov et al. (1994) have published predictions of possible observations for both eclipses and occultations between the major satellites: Colas (1995) describes the method to apply to observe these events by CCD 
cameras. Arlot \& Thuillot (1995), Thuillot (1995) and Thuillot \& Arlot (1995) give details on the organization of the next campaign PHESAT95. Stavinschi \& Bocsa (1995) and Zamarashkin et al. (1995) explain how they will organize these observations at Bucarest and Pulkovo observatories respectively. It is hoped that many observers will participate to the campaign PHESAT95, because if the timing of events is obtained at the precision level of some tenths of second, the expected precision on inter-satellite angular distance may be as good as 0 ".01 (that is $70 \mathrm{~km}$ on positions!).

Spatial observations exist already which may be used also more extensively: From the tracking data collected during the Pioneer and Voyager missions, Campbell \& Anderson (1989) have deduced new values for the constants of the gravity field of Saturn. In particular, their $J_{2}$ value $(0.016298 \pm 0.000010$, with an equatorial radius of $60300 \mathrm{~km})$ is now the most accurate determination.

\subsection{THEORIES}

Recent progress concern separately the theory of Hyperion and the theory of all other satellites:

For Hyperion, Message (1989) has proposed a new analytical theory of motion, using formal manipulations on computer to expand the disturbing function of the Titan-Hyperion problem in the neighbourhood of the libration. Second-order terms are computed for the long-periodic part of the motion, the influence of the short-period terms of first order being computed by semi-numerical quadratures. Message (1993) shows two fits of this theory to observations collected in the two periods 1875-1922 and 19681984 , giving two dynamically consistent sets of parameters and coefficients, one for each period; however, these two solutions are slightly different and not exactly compatible each with other, since in particular they correspond to two different values for the Titan's mass; that makes to much regret the total lack of observation of Hyperion during more than forty years, from 1923 to 1967.

Gerasimov \& Mushailov (1991) have also constructed an analytical resonant solution of the external case of the circular restricted 3-body problem and accounting for the Saturn's oblateness; however, being independent of the Titan's eccentricity, this solution does not include the important oscillations whose period is 18.8 years.

Other works on Hyperion try to represent numerical integrations by various functions of $t$, giving semi-analytical solutions:

Duriez (1992) gives a first synthetic theory of motion for Titan-Hyperion, obtained iteratively from the representation of Taylor et al. (1987) by substituting numerically a solution in the Lagrange equations and then by 
using a multivariable Fourier transformation to reconstruct trigonometrical developments with arguments as combinations of 3 fundamental angles (libration, pericenter and synodic angle). This process allows to construct extensively the long and short period terms in the planar problem, up to the kilometer level. Many new terms exceeding $500 \mathrm{~km}$ are found which were not present in Taylor et al. (1987). Some long-period perturbations of Titan by Hyperion are also found which would be non negligible at the 10 $\mathrm{km}$ level (assuming the Hyperion's mass equal to half that of Mimas).

Taylor (1992) gives another synthetic theory of motion for Hyperion, obtained from a numerical integration of Titan-Hyperion over \pm 25 years around 1973 and fitted to recent observations: Then, Taylor represent the resulting time-series by fitting to them, by least-square method, given series of trigonometrical terms with adjusted amplitudes and with arguments like in Taylor et al. (1987) or Duriez (1992). That allows to construct all longperiod terms greater than 0 ".07 (as seen from earth) and all short-period terms greater than $0^{\prime \prime} 02$. Compared to $1967-1983$ observations, such a representation gives r.m.s. residuals of $0^{\prime \prime} .28$ for the Titan-Hyperion intersatellite positions, that is comparable to residuals in Taylor et al. (1987).

In fact, the difficulty to lower the residuals comes from the very bad convergence of the short-period terms: Duriez (1994) gives a new synthetic theory for Hyperion including all long-period terms greater than $1 \mathrm{~km}$ and all short period term greater than $5 \mathrm{~km}$; more than 500 terms are thus computed but the maximum difference over one century between the numerical integration and its representation remains as large as about $300 \mathrm{~km}$ due essentially to the short period part of the solution. Unlike Duriez (1992), this new representation is constructed from a numerical integration of the 3D Titan-Hyperion problem, over 1500 years, with numerical filtering to obtain only long-term motions; then, a discriminating frequency analysis allows to represent the time-series as sums of long period terms (periods between $45 \mathrm{~d}$ and $700 \mathrm{y}$; after subtracting this representation from the unfiltered time-series the same method of frequency analysis allows to reconstruct short period terms (periods found between $3 \mathrm{~d}$ and $45 \mathrm{~d}$ ). Recently, partial derivatives of this representation with respect to masses and initial conditions have been computed, and a preliminary adjustment to recent observations has given r.m.s. residuals of $0^{\prime \prime} .22$ (not yet published).

At last, Kirsanov (1995) presents new elements of Hyperion as polynomials of $t$, obtained from a numerical integration of Titan-Hyperion, fitted to 1967-1981 observations; ten years from 1995 are described by series of polynomials, each one being valid over 300 days.

Concerning the other satellites, there are both a new theory of motions and new fits to ground-based observations:

Duriez \& Vienne (1991) and Vienne \& Duriez (1992) present a new 
TABLE 1. - r.m.s. residuals of some major sets of observations (in arcseconds) obtained in the 3 recent adjustments over one century by Dourneau (1993), by Harper \& Taylor (1993) [H\&T in the table] and by Vienne \& Duriez (1995) [TASS in the table].

\begin{tabular}{|c|c|c|c|c|}
\hline observations & number & Dourneau & $\mathrm{H} \& \mathrm{~T}$ & TASS \\
\hline Struve (1898) & 1780 & 0.15 & 0.14 & 0.12 \\
\hline USNO (1954) & 2520 & 0.23 & 0.22 & 0.20 \\
\hline Sinclair $(1974,1977)$ & 860 & 0.18 & 0.12 & 0.13 \\
\hline Pascu (1982) & 2330 & & 0.13 & 0.13 \\
\hline Aksnes \& al $(1984)^{(*)}$ & 14 & 0.025 & 0.018 & 0.015 \\
\hline Dourneau et al (1989) & 950 & 0.27 & 0.28 & 0.27 \\
\hline Veillet \& Dourneau (1992) & 300 & 0.19 & 0.18 & 0.11 \\
\hline Veillet \& Dourneau (1992) & 1400 & 0.11 & 0.13 & 0.12 \\
\hline all & 000 & 0.20 & 0.17 & 0.15 \\
\hline
\end{tabular}

(*) residuals taken from Viateau \& Rapaport (1995)

global and analytical theory of the motions of all major satellites (except Hyperion, seen above). The aim of this work is to give a coherent representation for the motions of all satellites, considered all together, in the J2000 reference system, with a precision compatible with spatial observations. The method is the same as in a general planetary theory: The Lagrange's equations are constructed analytically up to degree six in eccentricities and inclinations and to order 2 in the masses and in the oblateness coefficients of the Saturn's figure; all terms greater than $1 \mathrm{~km}$ have been retained in the short period perturbations as well as in the critical system which collects all secular, resonant and solar perturbations. Then, a numerical integration of this system is made over $1200 \mathrm{y}$ for the four inner resonant satellites (step $4 \mathrm{~d}$ ), and over $9000 \mathrm{y}$ for Rhea, Titan and Iapetus (step $100 \mathrm{~d}$ ); after that, frequency analysis is applied to the resulting time-series, allowing to represent the corresponding mean elements as sums of periodic terms whose arguments are then identified as integer combinations of some fundamental arguments. That leads finally to a semi-numerical representation of all orbital elements. The internal precision of this representation is only a few kilometers for all satellites except Iapetus (for which it is only $100 \mathrm{~km}$ over one century). At last, the partial derivatives of this representation with respect to all physical parameters and to initial conditions have also been computed; the resulting theory is called TASS. The main characteristic of this solution comes from the ability to produce a coherent determination of all dynamical parameters for the complete Saturn's system, because each coefficient, each frequency, each phase in the resulting series is expressed analytically with respect to the masses, to the oblateness coefficients and to the initial conditions of motions. 
TABLE 2. - r.m.s. residuals by satellites (in arcseconds), obtained by Dourneau (1993) and by Vienne \& Duriez (1995).

\begin{tabular}{cccccccc}
\hline & 1 & 2 & 3 & 4 & 5 & 6 & 8 \\
\hline Dourneau & 0.23 & 0.20 & 0.20 & 0.20 & 0.19 & 0.20 & 0.25 \\
TASS1.6 & 0.18 & 0.14 & 0.13 & 0.13 & 0.12 & 0.13 & 0.19 \\
\hline
\end{tabular}

Vienne \& Duriez (1995) present a fit of TASS to observations made between 1874 and 1989, and give formulas to compute ephemerides in the J2000 reference system. A few time before, Harper \& Taylor (1993) have produced a new fit of their theory to almost the same observations (from the Strugnell \& Taylor catalogue, completed by observations made by Pascu and by Veillet \& Dourneau). Hence, with Dourneau (1993), there are now 3 representations of the major satellites which have been adjusted to more than one century of observations.

Table 1 gives the main results from these three fits, showing that the r.m.s. residuals are now between $0^{\prime \prime} .20$ and $0^{\prime \prime} .15$ (for the set of all satellites except Hyperion). The 30000 observations concern TASS but there are 8000 observations more in Harper \& Taylor (analyzed in Harper \& Taylor 1994) and 8000 observations less in Dourneau. We see also that the old observations by Struve seem as good as those from Veillet \& Dourneau. The 14 observations from Aksnes concern mutual events made during the previous disappearance of the Saturn's rings in 1980 : The very small residuals, (computed by Rapaport 1995), reveal the great interest of such observations, and show that the theories of the satellites concerned by these observations (Enceladus to Rhea from Nov. 1979 to Apr. 1980) seem now as good as these residuals.

Table 2 gives the residuals obtained for each satellite : They are still greater for Mimas and Iapetus, requiring probably new developments for their theory. Moreover, Rapaport \& Le Campion (1995) have compared the three solutions given by Dourneau, Harper \& Taylor et Vienne \& Duriez in the 1960-2000 period, showing also that the differences between theories are the greatest for Mimas and Iapetus.

At last, Table 3 gives the values of some dynamical parameters obtained by adjustment of each theory to ground-based observations; there is a good agreement except for the mass of Enceladus from TASS, but this mass gives a mean density closer to 1 , like that of Mimas or Tethys. Finally, TASS appears to give now the smallest residuals and probable errors, but space observations would still improve the theories. 
TABLE 3. - Adjustments of some physical parameters, where C\&A = Campbell \& Anderson (1989) (from tracking data of Pioneer and Voyager missions), with in parenthesis the probable error expressed in the units of the last digits.

\begin{tabular}{crrrr}
\hline parameters $\times 10^{6}$ & \multicolumn{1}{l}{ Dourneau } & \multicolumn{1}{l}{ H\&T } & \multicolumn{1}{c}{ TASS } & \multicolumn{1}{c}{ C\&A } \\
\hline$m_{1}$ & $0.0648(21)$ & $0.0646(11)$ & $0.0634(8)$ & \\
$m_{2}$ & $0.206(55)$ & $0.213(46)$ & $0.069(21)$ & \\
$m_{3}$ & $1.088(31)$ & $1.076(18)$ & $1.060(13)$ & \\
$m_{4}$ & $1.954(58)$ & $1.916(36)$ & $1.963(21)$ & \\
$m_{5}$ & & & $4.320(380)$ & $4.059(53)$ \\
$m_{6}$ & & & & $236.368(8)$ \\
$m_{8}$ & $3.7(1.7)$ & & $3.10(50)$ & $2.79(8)$ \\
$J_{2}$ & $16326(54)$ & $16298(38)$ & $16285(5)$ & $16298(5)$ \\
\hline
\end{tabular}

\section{Conclusion}

A great deal of work has been spent to improve both theories of motion and ground-based observations of all satellites. For the major satellites, we can hope that the new theories will be improved soon by taking account of new observations of mutual events in 1995-1996. For the other small satellites, some progress has still to be made to obtain the same precision in both theories and observations. However, we must soon take up the challenge of the CASSINI mission, which will require theories able to represent the positions of all satellites with a precision perhaps better than a few kilometers. In effect, the spacecraft will be put in orbit around Saturn, and during the few years of the mission, several tens of close encounters with Titan will be necessary to produce, by gravitational assistance, all the various orbits needed to explore the all satellite's system; to plan these encounters, the motion of Titan will have to be known with a precision of a few kilometers. All that will be very important to know accurately the present state of all motions, that will then allow to extent them with confidence to past and future long-term evolution.

\section{References}

Aksnes K., Franklin F., Millis R., Birch P., Blanco C., Catalano S., Piironen J.: 1984, 'Mutual phenomena of galilean and saturnian satellites in 1973 and 1979/1980' Astron. J. 89, 280-286.

Aksnes K., Dourneau G.: 1994, 'Mutual events of Saturn's satellites in 1995-1996' Icarus 112, 545-548.

Arlot J.E., Thuillot W.: 1993, 'Eclipses and mutual events of the first eight saturnian satellites during the 1993-1996 period', Icarus 105, 427-440.

Arlot J.E., Thuillot W.: 1995, 'The former campaigns of observations of mutual events', to appear in the proceedings of the 'Atelier de travail PHESAT95', Bucarest, Sept. 1994. 
Beurle K., Harper D., Jones D.H.P., Murray C.D., Taylor D.B., Williams I.P.: 1993, 'Preliminary analysis of CCD observations of Saturn's satellites', Astron. Astrophys. 269, 564-567.

Beurle K.: 1994, 'CCD observations of Saturn's satellites', in Royal Greenwich Observatory Workshop: Galactic and solar system optical astrometry, p312-317.

Campbell, J.K., Anderson, J.D.: 1989, 'Gravity field of the saturnian system from Pioneer and Voyager tracking data', Astron. J. 97, 1485-1600.

Colas F.: 1991, 'Nouvelles observations CCD astrométriques pour l'étude dynamique des satellites des planètes: Application au mouvement de satellite Thebe de Jupiter' Thesis, Paris Observatory.

Colas F.: 1995, 'Observation CCD des phénomènes des satellites de Saturne', to appear in the proceedings of the 'Atelier de travail PHESAT95', Bucarest, Sept. 1994.

Dermott S.F., Malhotra R., Murray C.D.: 1988, 'Dynamics of the Uranian and Saturnian Satellite System: a chaotic route to melting Miranda ?', Icarus 76, 295-334.

Dourneau G.: 1987, 'Observation et étude du mouvement des huit premiers satellites de Saturne', Dr.thesis, Université de Bordeaux I, Bordeaux.

Dourneau G.: 1993, 'Orbital elements of the eight major satellites of Saturn determined from a fit of their theories of motion to observations from 1886 to 1985', Astron. Astrophys. 267, 292-299.

Duriez L.: 1992, 'A synthetic theory of motion for Titan-Hyperion', in IAU Symposium 152: Chaos, resonance and collective dynamical phenomena in the solar system p209214.

Duriez L., Vienne A.: 1991, 'A general theory of motion for the eight major satellites of Saturn. I : Equations and method of resolution', Astron. Astrophys. 243, 263-275.

Duriez L., Vienne A.: 1994, 'Modélisation du mouvement d'Hyperion', in 'Notes Scientifiques et Techniques du Bureau des Longitudes', S50, 115-120.

Emel'yanov N.V., Gasanov S.A., Nasonova L.P.: 1994, 'Mutual events in Saturn's satellite system from 1995 to 1996 ' Astron. Reports 38, 708-717.

Gerasimov V.P., Mushailov B.R.: 1991, 'Evolution of orbits of Hyperion', Sov. Astron. 35 202-205.

Harper D., Taylor D.B., Sinclair A.T.: 1989, 'Analysis of the orbits of Titan Hyperion and Iapetus by numerical integration', Astron. Astrophys. 221, 359-365.

Harper D., Taylor D.B.: 1993, 'The orbits of the major satellites of Saturn', Astron. Astrophys. 268, 326-349.

Harper D., Taylor D.B.: 1994, 'Analysis of ground-based observations of the satellites of Saturn 1874-1988', Astron. Astrophys. 284, 619-628.

Harper D.: 1995, 'Astrometry of Saturn's satellites from La Palma' to appear in the proceedings of the 'Atelier de travail PHESAT95', Bucarest, Sept. 1994.

Kirsanov N.O.: 1995, 'A theory of the motion of Hyperion' to appear in the proceedings of the 'Atelier de travail PHESAT95', Bucarest, Sept. 1994.

Le Campion J.F., Geffert M., Dulou M.R., Colin J.: 1992 'An astrometric catalogue of stars in the region of M15', Astron. Astrophys. Sup. Ser. 95, 233-247.

Le Floch J.C.: 1995, 'L'observation des satellites de Saturne à l'observatoire de Bordeaux' to appear in the proceedings of the 'Atelier de travail PHESAT95', Bucarest, Sept. 1994.

Message J.P.: 1989, 'The use of computer algorithms in the construction of a theory of the long-period perturbations of Saturn's satellite Hyperion' Celest. Mech. 45, 45-53.

Message J.P.: 1993 'On the second order long-period motion of Hyperion', Celest. Mech. $56,277-284$.

Pascu D., Schmidt R.E.: 1990, 'Photographic positional observations of Saturn' Astron. J. 99, 1974-1984.

Pascu D.: 1995, 'CCD observations of planetary satellites at the U.S. Naval Observatory', to appear in the proceedings of the 'Atelier de travail PHESAT95', Bucarest, Sept. 1994. 
Rapaport M., Le Campion J.F.: 1995, 'Observations CCD du système des satellites de Saturne faites à Bordeaux. Comparaisons avec les théories' to appear in the proceedings of the 'Atelier de travail PHESAT95', Bucarest, Sept. 1994.

Rohde J.R., Pascu D.: 1993, 'Astrometric observations of Helene, Telesto and Calypso' Bull. Amer. Astron. Soc. 25, 1235.

Rohde J.R., Pascu D.: 1994, 'CCD Astrometry of Helene, Telesto and Calypso: 1993 observations' Bull. Amer. Astron. Soc. 26, 1024.

Sessin W.: 1992, 'Hori auxiliary system for Mimas-Tethys' in IAU Symposium 152: Chaos, resonance and collective dynamical phenomena in the solar system p223-226.

Sinclair A.T.: 1974, 'A theory of the motion of Iapetus', Month. Not. Roy. Astrom. Soc. 169, 591-605.

Sinclair A.T.: 1977, 'The orbits of Tethys, Dione, Rhea, Titan and Iapetus', Month. Not. Roy. Astrom. Soc. 180, 447-460.

Sinclair A.T., Taylor D.B.: 1985, 'Analysis of the orbits of Titan, Hyperion, and Iapetus by numerical integration and by analytical theories', Astron. Astrophys. 147, 241246.

Soma M.: 1992, 'Eclipses of Iapetus in 1993', Astron. Astrophys. 265, L21-L24.

Stavinschi M., Bocsa G.: 1995, 'The Romanian tradition in the observations of Jupiter's and Saturn's satellites', to appear in the proceedings of the 'Atelier de travail PHESAT95', Bucarest, Sept. 1994.

Strugnell P.R. Taylor D.B.: 1990, 'A catalog of ground-based observations of the eight major satellites of Saturn 1874-1989' Astron. Astrophys. Sup. Ser. 83, 289-300.

Taylor D.B., Shen K.X.: 1988, 'Analysis of astrometric observations from 1967 to 1983 of the major satellites of Saturn', Astron. Astrophys. 200, 269-278.

Taylor D.B., Sinclair A.T., Message P.J.: 1987, 'Corrections to the theory of the orbit of Saturn's satellite Hyperion' Astron. Astrophys. 181, 383-390.

Taylor D.B., Morrison L.V., Rapaport M.: 1991, 'Saturn's position derived from meridian circle observations of Titan and Iapetus' Astron. Astrophys. 249, 569-573.

Taylor D.B.: 1992, 'A synthetic theory for the perturbations of Titan on Hyperion', Astron. Astrophys. 265, 825-832.

Thuillot W.: 1995, 'Les éphémérides pour les observateurs PHESAT95', to appear in the proceedings of the 'Atelier de travail PHESAT95', Bucarest, Sept. 1994.

Thuillot W., Arlot J.E.: 1995, 'La prédiction des phénomènes des satellites de Saturne pour la période de 1995', to appear in the proceedings of the 'Atelier de travail PHESAT95', Bucarest, Sept. 1994.

Veillet C., Bois E., Oberti P.: 1988, ' 12 ans d'astrométrie photographique des satellites de Saturne, Uranus et Neptune', journées de planétologie, 457-460.

Veillet C., Dourneau G.: 1992, '1980-1985 astrometric observations of the first eight satellites of Saturn at Pic-du-Midi, ESO and CFH', Astron. Astrophys. Sup. Ser. 94, 291-297.

Viateau B., Rapaport M.: 1995, 'Observations astrométriques à l'Observatoire de Bordeaux', in the proceedings of the IAU symposium 172, Paris July 1995.

Vienne A., Duriez L.: 1991, 'A general theory of motion for the eight major satellites of Saturn. II : Short-period perturbations', Astron. Astrophys. 246, 619-633.

Vienne A., Duriez L.: 1992, 'A general theory of motion for the eight major satellites of Saturn. III : Long-period perturbations', Astron. Astrophys. 257, 331-352.

Vienne A., Duriez L.: 1995, 'TASS1.6: Ephemerides of the major saturnian satellites', Astron. Astrophys. 297, 588-605.

Zamarashkin K.N., Kirsanov N.O., Kisseleva T.P., Kisselev A.A., Batrakov J.V.: 1995, ' $C C D$ astrometric observations of the saturnian satellites system on the 26-inch refractor at Pulkovo to be made in 1995-1996 campaign and their comparison with parallel photographic observations', to appear in the proceedings of the 'Atelier de travail PHESAT95', Bucarest, Sept. 1994. 